\title{
FORMACIÓN PARA EL EMPRENDIMIENTO CASO DE ESTUDIO INSTITUTO SUPERIOR TECNOLÓGICO BOLIVARIANO DE TECNOLOGÍA (ITB), ECUADOR*
}

\section{ENTREPRENEURSHIP TRAININGCASE STUDY BOLIVARIAN HIGHER TECHNOLOGICAL INSTITUTE OF TECHNOLOGY (ITB), ECUADOR}

Recibido: 6 de diciembre de 2019

Evaluado: 13 de febrero de 2020

Aprobado: 12 de mayo de 2020

\author{
Roxana Chiquito** \\ Instituto Superior Tecnológico Bolivariano de Tecnología, Ecuador \\ Orcid: https://orcid.org/0000-0002-1650-8456 \\ Mireya Zuñiga ${ }^{* * *}$ \\ Instituto Superior Tecnológico Bolivariano de Tecnología, Ecuador \\ Orcid: https://orcid.org/0000-0002-4458-5771

\section{Karen Ruiz ${ }^{* \star \star}$} \\ Instituto Superior Tecnológico Bolivariano de Tecnología, Ecuador \\ Orcid: https://orcid.org/0000-0003-0728-722X
}

Cómo citar este artículo: Chiquito, R., Zuñiga, M., Ruiz, K. (2020). Formación para el emprendimiento. Caso de estudio Instituto Superior Tecnológico Bolivariano de Tecnología (ITB), Ecuador. Revista Estrategia Organizacional, 9(2). doi: https://doi.org/10.22490/25392786.4044

* $\quad$ Artículo de investigación desarrollado en Ecuador en el Instituto Superior Tecnológico Bolivariano de Tecnología

** PhD. Ingeniera en Sistemas Administrativos Computarizados, Doctora en Ciencias Pedagógicas, Docente a tiempo completo del Instituto Superior Tecnológico Bolivariano de Tecnología, Directora del Proyecto de Vinculación Incubadora de Emprendimiento. Correo electrónico: rchiquito@bolivariano.edu.ec

*** PhD. Ingeniera en Sistemas Administrativos Computarizados, Magíster en Gerencia Educativa, Doctora en Ciencias Pedagógicas, Docente a tiempo completo del Instituto Superior Tecnológico Bolivariano de Tecnología, Acreditada por la SENESCYT para realizar actividades de investigación en el Ecuador. Correo electrónico: szuniga@bolivariano. edu.ec

**** Magíster en Finanzas y Proyectos Corporativos, Ingeniera en Sistemas Administrativos Computarizados, Docente a tiempo completo del Instituto Superior Tecnológico Bolivariano de Tecnología. 


\title{
RESUMEN
}

Introducción: La presente investigación está relacionada con la formación para el emprendimiento en el Ecuador, tomando como referencia un caso de estudio del Instituto Superior Tecnológico Bolivariano de Tecnología. Las condiciones generales para promover el emprendimiento en el país, han mejorado considerablemente en los últimos años, en la actualidad existen diversos programas de financiamiento para jóvenes emprendedores a nivel municipal y nacional. Metodología: Se plantea dos aspectos que se relacionan a la formación del emprendedor, que son la formación para el emprendimiento-proyectos de vida/profesionales, que responde a la autorrealización del ser humano a través del cumplimiento de sus objetivos personales y profesionales, también se considera la formación para el emprendimiento-educación de la creatividad que se relaciona con la esencia trasformadora del ser humano. Resultados: Para el emprendimiento del tecnólogo en administración de empresas se constituye la modelación del proceso de formación de la competencia emprendimiento, a través de tres etapas: planeación de la formación para el emprendimiento, intervención formativa del emprendimiento y evaluación de la formación para el emprendimiento. Conclusiones: El desarrollo de la formación para el emprendimiento en sus tres etapas se despliegan tres dimensiones: dimensión curricular, dimensión extracurricular y la dimensión socio-comunitaria. Estas dimensiones recorren un camino que va desde la formación académica, hasta la modelación de la gestión de empresas. Así la interrelación de las etapas y dimensiones del proceso de formación para el emprendimiento permite la formación del futuro tecnólogo en administración de empresas para el emprendimiento a partir de la apropiación de la competencia emprendimiento.

Palabras claves: formación, competencias, emprendimiento, administración.

\begin{abstract}
Introduction: This research is related to the formation of entrepreneurship in Ecuador, taking as a reference a case study of the Instituto Superior Tecnológico Bolivariano de Tecnología. The general conditions to promote entrepreneurship in the country have improved considerably in recent years, there are currently various financing programs for young entrepreneurs at the municipal and national levels. Methodology: There are two aspects relate to the training of the entrepreneur, which are the training for entrepreneurship-life / professional projects, which responds to the self-realization of the human being through the fulfillment of their personal and professional objectives, it is also considered the training for entrepreneurship-education of creativity that
\end{abstract}


relates to the transforming essence of the human being. Results: For the entrepreneurship of the technologist in business administration the modeling of the training process of the entrepreneurial competence is constituted, through three stages: planning of the training for entrepreneurship, formative intervention of entrepreneurship and evaluation of training for entrepreneurship. The development of training for entrepreneurship in its three stages unfolds three dimensions: curricular dimension, extracurricular dimension and the socio-community dimensión. These dimensions cover a path that goes from academic training, to business management modeling. Conclusions: The interrelation of the stages and dimensions of the training process for entrepreneurship allows the formation of the future technologist in business administration for entrepreneurship from the appropriation of entrepreneurship competence.

Keywords: Training, Skills, Entrepreneurship, Management.

\section{INTRODUCCIÓN}

El crecimiento económico, es uno principales objetivos de las naciones, por ende, es de vital importancia promover iniciativas que contribuyan a la generación de empleos y oportunidades de desarrollo económico y social de sus habitantes.

Los jóvenes cumplen un rol fundamental en el cumplimiento de este objetivo, la falta de experiencia, de recursos y de una red de apoyo ha sido siempre una barrera para impulsar el desarrollo de emprendedores, por ende, las políticas gubernamentales buscan la articulación de instituciones de educación, investigación y la comunidad empresarial.

Según el informe de Lasio, Ordeñana, Caicedo, Sarmiento, y Izquierdo. (2018), "establece tres factores que limitan el emprendimiento, cuya puntuación está bajo el promedio de los países de la región, estos factores son: políticas gubernamentales, apoyo financiero y la capacidad para emprender". 
Además, se identificaron problemas asociados a condiciones marco como acceso a información disponible, educación y entrenamiento, evidenciando su incidencia en la capacidad para emprender más allá de las habilidades innatas del emprendedor.

Los mismos autores señalan que en Ecuador, el $51 \%$ de la población adulta consideran iniciar un negocio en los próximos 3 años, pero de estos, tan solo el 21\% realiza alguna acción para realmente iniciarlo, uno de los factores que incide es el miedo al fracaso, ya que este paraliza a los emprendedores y limita sus oportunidades para luchar por sus metas.

Para el desarrollo del ecosistema emprendedor es imprescindible el aporte de las instituciones de educación superior a través de la formación de profesionales integrales que generen e inicien negocios creadores de nuevos puestos de trabajo.

Además, del establecimiento de alianzas con otras organizaciones públicas y privadas, permitiendo de esta manera adaptar los modelos existentes a la realidad educativa y nacional. Por ello, es importante generar espacios para la creación y desarrollo de emprendimientos, así como acceder a herramientas, recursos, contactos, capacitaciones y relaciones que se necesitan para crecer y desarrollar su capacidad de gestión en el mercado local, nacional e internacional, es vital para catapultar iniciativas mediante la generación de una nueva perspectiva y actitud empresarial, desde el punto de vista de la innovación.

Este documento describe los elementos teóricos y relaciones implícitas en la formación para el emprendimiento de tecnólogos en el Instituto Superior Tecnológico Bolivariano de Tecnología (ITB), institución de educación superior que trabaja constantemente en el diseño de estrategias educativas a fin de dar respuesta a nuevas necesidades relacionadas al empleo, dando oportunidades a los estudiantes, graduados y comunidades, en general de cambiar la empleabilidad por la emprendibilidad además de considerar el emprendimiento como un valor compartido de la institución y se definiéndose como social y solidario.

De ahíque, en Ecuador se hace urgente establecer una concepción pedagógica de formación para el emprendimiento, las relaciones y etapas necesarias para formar profesionales emprendedores, con el fin de desarrollar las potencialidades del país en el tema. Cuestión que el ITB asume entre sus prioridades. 
https://doi.org/10.22490/25392786.4044

\section{METODOLOGÍA}

El Instituto Superior Tecnológico Bolivariano de Tecnología (ITB), como institución de educación comprometida con el desarrollo económico y social del país, trabaja constantemente en el diseño de estrategias educativas a fin de dar respuesta a nuevas necesidades relacionadas al empleo, dando oportunidades a los estudiantes, graduados y comunidad general de cambiar la empleabilidad por la emprendibilidad.

Tolozano, Ferrer, y Forgas (2016), proponen un modelo pedagógico de gestión educativa para el ITB en el que el emprendimiento se considera un valor compartido de la institución y se define como social y solidario.

En el marco del modelo pedagógico antes mencionado se desarrolló una concepción pedagógica de formación para el emprendimiento, como un sistema de ideas y conceptos relacionados de manera esencial y estable, estructurado sobre la base de presupuestos de partida que aportan el marco referencial para el análisis, la representación y la concreción en la práctica pedagógica del proceso de formación, la misma permite orientar el proceso de formación del profesional desde la educación en valores sobre la base de una concepción ética del emprendimiento como humanista y sostenible, contribuyendo al logro de un desempeño profesional emprendedor en las condiciones de la sociedad ecuatoriana. (Chiquito y Corton, 2016)

Para el desarrollo de esta concepción se hace necesario analizar los referentes teórico para la formacion para el emprenidmiento y el emprendimiento.

En la literatura aparecen diversos términos para referirse al emprendimiento, Nueno, (2005), señala que "el emprendedor empresario, es el que a su vez se puede dividir en el emprendedor relacionado con la creación de nuevos negocios y el intraemprendedor, definido como el directivo que crea un negocio al interior de su empresa". Este autor se refiere a dos aristas del emprendimiento que se tienen en consideración, aunque limita el emprendimiento a la actividad empresarial.

Según Samaniego (2011), en "Ecuador el concepto emprendimiento, incluye en la última década la categoría social a pesar que desde su concepción mantiene el concepto de creación y/o innovación de una empresa nueva o que ya existe, como agente dinámico e innovador en el 
desarrollo". Se concuerda con este criterio, pero se considera que aun predomina la concepción de que el emprendedor es solo el creador de empresas y la dimensión social no es suficientemente desarrollada.

Bucardo, Saavedra, y Camarena (2015), distinguen entre los términos de emprendedor y empresario; según ellos el emprendedor utiliza el ingenio, la audacia, el entusiasmo, la ilusión y la insatisfacción laboral para realizar un cambio a través de la innovación de las ideas, productos o procesos, sin que esto implique necesariamente su participación en la creación o dirección de las empresas; el empresario invierte en la empresa capital propio o ajeno y la dirige con la finalidad de obtener un rendimiento, se comprende esta posición aunque se advierte la necesidad de que ambos(el emprendedor y el empresario) confluyan en una misma persona.

Al referirse al emprendimiento los autores consultados asumen diversas posiciones entre las que predomina una visión economicista y empresarial. La tendencia economicista del emprendedor, infiere que su formación y capacitación se relaciona fundamentalmente con aspectos de índole económica y se refiere a los conocimientos y habilidades que se necesitan para desempeñarse como líderes en las empresas.

Según Tobon (2013) el emprendimiento es "planear, iniciar y sacar adelante proyectos hasta lograr unas determinadas metas, buscando la resolución de problemas del contexto y actuando con pertinencia, creatividad, eficacia y eficiencia", este autor vincula el emprendimiento a la solución de problemas del contexto en un espectro que va más allá de lo netamente económico y establece el vínculo entre emprendimiento y creatividad. Se tienen en cuenta estas ideas en el desarrollo de la investigación.

Echerman (2011), considera como emprendimiento a toda acción social, por la cual los individuos demuestran competencias para identificar oportunidades (expresadas en problemas, necesidades o carencias) en cualquier ámbito, deciden voluntariamente hacerse cargo de las mismas y gestionan diversos recursos con la finalidad de desarrollar soluciones de valor, éticas, factibles y perdurables.

Este criterio que también se considera, señala los diferentes momentos de una acción emprendedora y hace énfasis en el valor fundamentalmente ético de las soluciones de los problemas en el contexto. 
Desde las perspectivas estudiadas las autoras consideran que el emprendimiento a los efectos de la formación de los tecnólogos, se entiende como emprendimiento humanista y sostenible, significando la relación dialéctica entre los intereses individuales y sociales.

Esto significa que el emprendedor que se necesita formar no pretende obtener ganancias a toda costa sino obtener ganancias y progresar teniendo en cuenta el interés social, el respeto a los demás y la utilización de medios honrados y considerando el beneficio al hombre como el principal indicador de éxito en su actividad emprendedora. Esto fundamenta que la formación humanista y la educación ambiental constituyan temas transversales en el proceso de formación para el emprendimiento.

En el informe publicado por el foro económico mundial en el 2018, que mide como el país utiliza sus recursos y capacidad para proveer a sus habitantes de un alto nivel de prosperidad, Ecuador obtiene un puntaje 55.85 en el índice de competitividad que lo ubica en el puesto 86 , de los 140 países analizados, acorde a las potencialidades del país, estas cifras pueden mejorar. (DATOSMACRO, 2018)

Por ende, es imprescindible que las instituciones de educación brinden herramientas para enfrentar al fracaso considerándolo necesario para alcanzar las metas propuesta ya que en Ecuador, desde la educación básica y media aún están regida por métodos establecidos que no incentivan la creatividad e innovación. La clave reside en no buscar el fracaso, pero tampoco temerlo: asumirlo como parte del viaje, por lo que es vital contar con escudo interno para enfrentar las críticas y el miedo al fracaso.

La Actividad Emprendedora Temprana (TEA), en el 2017 fue de 29.6\%, este porcentaje está conformado por el $19.9 \%$ de emprendedores naciente, siendo este el porcentaje de la población adulta que está en el proceso de iniciar un negocio y $9.6 \%$ que ya es dueño o administrador de un negocio nuevo, es decir, que ha estado en marcha durante menos de 42 meses. De estos, el 53\% de TEA en el Ecuador en el 2017 corresponde a adultos con edades entre 18 y 34 años. (Lasio, Ordeñana, Caicedo, Sarmiento, y Izquierdo, 2018) 
En años recientes, se considera que las condiciones generales para promover el emprendimiento en el país, han mejorado considerablemente, en la actualidad existen diversos programas de financiamiento para jóvenes emprendedores a nivel municipal y nacional, por tanto el escenario social y político comienza a ser favorable. $Y$ en la educación superior el emprendimiento no alcanza a todos los estudiantes ya que el contenido no constituye un eje transversal o asignatura de carácter obligatorio en todas las carreras, sino que se imparten con frecuencia como parte de materias opcionales. Ya que generalmente se asocia al emprendimiento con las profesiones relacionadas con las ciencias administrativas o negocios. Además, es necesario implementar métodos que favorecen la experiencia de los estudiantes fuera del aula y su conexión con el mundo real, esto es fundamental para formar emprendedores.

Importante en la formación para el emprendimiento resuelva determinar las relaciones socio -educativas en que se debe desarrollar el PEA, para determinarlos se utilizaron métodos teóricos, empíricos y matemáticos, entre los teóricos el análisis-síntesis y la inducción- deducción para el procesamiento de la información contenida en los textos consultados, en los documentos normativos del proceso de formación y en los datos arrojados por el diagnóstico; la elaboración de los aportes; y la redacción del informe de investigación.

El método histórico-lógico se utilizó en la sistematización de los referentes teóricos y metodológicos.

Entre los métodos empíricos se utilizó el análisis documental para procesar la información contenida en los documentos que norman el proceso de formación. La observación de actividades de la carrera para constatar cómo se aborda en la práctica pedagógica el proceso de formación y específicamente la formación para el emprendimiento.

Se utilizaron técnicas empíricas como entrevistas a los docentes y encuestas a egresados y estudiantes de la carrera para diagnosticar el estado actual del proceso de formación para el emprendimiento desde la perspectiva de ambos actores.

Para valorar la efectividad del aporte, se utilizó el criterio de especialistas a través de taller de socialización y la aplicación parcial de la propuesta a través de la investigación acción. 
Además, entre los métodos matemáticos se utilizó el método estadístico para el procesamiento de los resultados del diagnóstico. En el desarrollo de la investigación se realizó la triangulación de fuentes y de métodos, como metodología arribando a una propuesta parcial para la solución del problema.

\section{RESULTADOS}

\section{RELACIONES PARA LA FORMACIÓN DE EMPRENDEDORES EN EL PEA DEL ITB.}

Con bases en el enfoque ético y desde la formación por competencias de la formación para el emprendimiento se connota como una perspectiva teórica para el análisis de este proceso y las relaciones necesarias para la formación de profesionales emprendedores, son las siguientes:

\section{Formación para el emprendimiento-motivación profesional.}

La formación para el emprendimiento requiere potenciar en el individuo la capacidad de elección y toma de decisiones lo que depende directamente del deseo, la intención de realizar un acto determinado, es por ello que la autovaloración interviene como un componente importante a desarrollar en la personalidad.

Potenciar la autovaloración constituye un elemento esencial, al ser esta, un elemento decisivo en el comportamiento emprendedor. La autovaloración estructurada adecuadamente, deviene un factor importante para desarrollar un acto de emprendimiento, cuando se establecen las adecuadas relaciones entre los componentes cognitivos, afectivos y volitivos de la personalidad, expresados en la flexibilidad o capacidad de asumir elementos disonantes con la propia identidad personal e integridad.

El desempeño profesional emprendedor requiere de una elevada motivación, específicamente hacia la profesión que se convierta en fuerza motriz de la acción emprendedora sobre la base de aspectos cognitivos, afectivos y volitivos. 


\section{Formación para el emprendimiento-orientación educativa (profesional).}

La formación para el emprendimiento supone una relación de orientación, de ayuda del orientador profesional (docente) y el resto de los miembros de la comunidad educativa (empresarios y trabajadores vinculados a la práctica pre profesional, familiares) con el orientado (estudiante) en las diferentes etapas, dimensiones y contextos del proceso de su formación, con el objetivo de propiciar las condiciones necesarias para el desarrollo de las potencialidades de la personalidad del estudiante que le posibiliten asumir un modo de actuación emprendedor en el proceso de su formación y en su desempeño profesional futuro.

Esta ayuda no se limita a brindar información, sino que implica un proceso de educación de la personalidad a través de los métodos de la labor educativa. Significa facilitar el reconocimiento de los problemas para los que el estudiante necesita la ayuda-en este caso la formación para el emprendimiento-, y la búsqueda conjunta (orientador/orientado) de alternativas para su solución.

\section{Formación para el emprendimiento-proyectos de vida/profesionales.}

Los proyectos profesionales son parte de los proyectos de vida e integran orientaciones vitales de la personalidad, asociadas a la formación y desempeño del sujeto en una profesión.

Estos se construyen en una etapa superior del desarrollo de la personalidad, y son expresión del grado de autodirección, autovaloración y autodeterminación del sujeto, para proyectar su futuro profesional, trazándose metas, resultantes de sus potencialidades y capacidades para buscar alternativas de solución a los problemas profesionales que se les presentan en su formación y desempeño; determinando las direcciones de los estudiantes, hacia el contenido de la profesión, al darse cuenta de quién es, qué quiere ser y en quién puede llegar a convertirse y cómo lograr sus propósitos profesionales.

La formación para el emprendimiento debe tener en cuenta potenciar los conocimientos, habilidades y valores; y las cualidades de la personalidad que permitan a los estudiantes identificar oportunidades de negocios y llevarlas a la práctica para lo cual deberán incorporar el emprendimiento a sus proyectos de vida /profesionales. 


\section{Formación para el emprendimiento-educación de la creatividad.}

En la creatividad se expresa la esencia transformadora del hombre, esta es característica de todos los individuos; un individuo creativo se caracteriza por cualidades como originalidad, curiosidad, pensamiento divergente, espontaneidad, flexibilidad, sensibilidad, productividad, libertad, perseverancia, capacidad para descubrir lo nuevo, de ver nuevas relaciones, temeridad para desafiar lo complejo, capacidad para reestructurar las cosas, eficacia, tenacidad, gusto por el cambio, independencia; cualidades que se identifican en esencia con las de un individuo emprendedor, de ahí la relación emprendimiento creatividad.

El emprendimiento exige cualidades de la personalidad que son al mismo tiempo rasgos que tipifican a la personalidad creativa. No existen modelos para desarrollar el pensamiento creador pues esto negaría su propia esencia; pero se pueden propiciar condiciones que lo favorezcan en el proceso pedagógico; para ello hay que tener en cuenta lo cognitivo y lo afectivo, ahí que la educación en la creatividad se considere tema transversal del proceso de formación para el emprendimiento.

La formación para el emprendimiento en las condiciones de Ecuador constituye una vía para articular la formación profesional con el desempeño ciudadano responsable y en consonancia con el progreso individual y colectivo, que constituyen objetivos estratégicos del desarrollo económico. Este proceso posibilita una interacción constructiva con la realidad, específicamente con la realidad socio productiva.

Estas relaciones a los efectos del proceso de formación discurren transversalmente en tres etapas del núcleo de la concepción pedagógica de formación para el emprendimiento:

- Planeación de la formación para el emprendimiento: incluye tres momentos el diagnóstico de preparación de docentes, directivos y empresarios para desarrollar el proceso de formación para el emprendimiento, la proyección contextualizada del mismo y la gestión de los recursos materiales y humanos necesarios para su desarrollo. 
- Intervención formativa del emprendimiento: el diagnóstico integral de estudiantes con énfasis en las cualidades y jerarquía de valores asociados al emprendimiento, la sensibilización y la participación emprendedora formativa.

- Evaluación de la formación para el emprendimiento: se evalúa contenidos, modos de actuación emprendedores y desempeño profesional emprendedor.

El desarrollo de la formación para el emprendimiento en sus tres etapas se despliega en las tres dimensiones, todas las etapas del proceso de formación se desarrollan en estas dimensiones, al mismo tiempo al interior de las etapas y las dimensiones existen relaciones de coordinación y complementación, las dimensiones son:

- Dimensión curricular

- Dimensión extracurricular

- Dimensión socio- comunitaria

Las etapas del proceso la planeación de la formación para el emprendimiento permite crear las condiciones para la intervención formativa del emprendimiento, etapa durante la cual se pueden introducir ajustes en la planeación, la evaluación de la formación para el emprendimiento se desarrolla durante todo el proceso pero adquiere peculiaridades en sus diferentes momentos y dimensiones, por ende, es indispensable establecer alguna aclaraciones en las dimensiones cuando transcurren por la etapa de evaluación de la formación para el emprendimiento.

La dimensión curricular incluye, formación académica emprendedora: parte de la concepción del pensum de la carrera Tecnología en Administración de Empresas, en el que se planifican y organizan los contenidos académicos que brindan los conocimientos, habilidades y valores necesarios para el emprendimiento los cuales se contextualizan en los syllabus de cada asignatura y en su correspondiente proceso de enseñanza aprendizaje. 
Su desarrollo incluye una variedad de actividades formativas profesionales sistematizadas desde las clases, talleres, proyectos empresariales, tareas de investigación, visitas a empresas, pasantías, entre otras. Éstas deben estar convenientemente concebidas, diseñadas, planifıcadas y ejecutadas y sobre todo intencionadas predominantemente desde lo formativo.

Para la formación para el emprendimiento, resulta necesario el enfoque integrador e interdisciplinar de la concepción y la instrumentación de currículo.

El tratamiento del contenido de la enseñanza desde esta dimensión deberá tener en cuenta:

- Su orientación profesional

- El aprendizaje activo y colaborativo

- La solución de problemas profesionales

- La flexibilidad en el desarrollo del proceso enseñanza aprendizaje

- La atención a la diversidad

En la dimensión curricular las acciones a desarrollar deberán concebirse e implementarse teniendo en cuenta como referente y como indicadores para la evaluación de su efectividad los elementos de la competencia emprendimiento y el contenido de los valores asociados al emprendimiento. Los resultados de esta evaluación deberán ser observables, medibles que se puedan evaluar en las evaluaciones de las diferentes asignaturas y en los modos de actuación del estudiante, como modelación del futuro desempeño profesional emprendedor.

Formación investigativa emprendedora: parte de la concepción del pensum y se orienta al desarrollo de habilidades investigativas y a la aprehensión del sistema de valores propios de la ética del estudiante a través de la adquisición de conocimientos relacionados con la política económica de la sociedad ecuatoriana, problemas de la producción y de los servicios nacionales 
y locales, documentos que regulan la creación y administración de empresas, necesidades y tendencias del mercado laboral, y otros.

La formación investigativa emprendedora deberá tener en cuenta:

- El papel de la investigación en la solución de problemas nacionales, locales y comunitarios.

- El vínculo de teoría con la práctica.

- El vínculo investigación-innovación.

- La interdisciplinaridad, aprovechando específicamente las potencialidades de los enfoques investigativos que aportan ciencias sociales como la sociología, la antropología, etc.

- La utilización de métodos de investigación integradores de lo cuantitativo y lo cualitativo.

- La socialización a través de las diferentes vías de los resultados de las investigaciones.

Formación práctica pre profesional emprendedora: esta formación implica la planificación de la práctica pre profesional de manera que se garantice el contacto de los estudiantes con empresas y empresarios que constituyan paradigmas del emprendimiento a nivel nacional, local o comunitario para aprovechar las potencialidades educativas de estas experiencias positivas, supone la sensibilización y capacitación de dichos empresarios sobre la base de su compromiso con la transformación de la realidad económica y el establecimiento de vínculos entre la institución educativa y las empresas sustentados en el propósito común de contribuir a la formación de los estudiantes para el emprendimiento.

La dimensión extracurricular incluye actividades no contempladas en la malla curricular pero que contribuyen al logro de los objetivos propuestos en el pensum y los syllabus de las diferentes asignaturas, desde las cuales se orienta y organiza el desarrollo de dichas actividades. Incluye in- 
vestigaciones, proyectos, ferias de innovación y/o de emprendimiento que refuerzan la formación. Su contexto de desarrollo puede ser la institución educativa, las empresas con las que se vincula la institución o las comunidades.

Las acciones desarrolladas en esta dimensión tienen un carácter eminentemente innovador e inciden de manera directa en la educación de la creatividad fomentando el trabajo en equipo, la cooperación y la responsabilidad. En las acciones de esta dimensión el estudiante dará solución a problemas profesionales hipotéticos o modelados formulados como tareas docentes y extradocentes.

La dimensión extracurricular, corresponde a la identificación de experiencias empresariales emprendedoras: este proceso orientado por los docentes le permitirá a los estudiantes sobre la base de determinados criterios en el contexto nacional, local y/o comunitario identificar empresas exitosas y no exitosas, y analizar críticamente los factores que inciden en los resultados, enfatizando en el papel y el lugar del emprendimiento en el logro de los mismos; como base para desarrollar debates, presentaciones y otras actividades que les permitan incorporar buenas prácticas empresariales en su formación.

Como criterios de identificación se sugieren:

- Resultados económicos ascendentes y estables

- Presentación de nuevos productos y servicios que resuelvan necesidades crecientes de la sociedad.

- Desarrollo de estrategias de comercialización creativa y efectiva.

- Innovación en la gestión empresarial.

La planeación de proyectos de innovación: estos proyectos no poseen gran nivel de complejidad, generalmente se desarrollan en equipos y tienen como propósito fundamental lograr que los estudiantes planeen e instrumenten soluciones a situaciones profesionales o no que les permiten 
desarrollar habilidades para identificar y solucionar problemas de la realidad que los entrenen para su futuro desempeño profesional.

Su realización es orientada desde las diversas asignaturas de la malla curricular, aunque con un enfoque interdisciplinario. La concepción de los proyectos deberá evidenciar un modo de actuación emprendedor.

La dimensión socio comunitaria, por su esencia es extracurricular pero se connota de manera particular por la significación que tiene para incorporar a los estudiantes a la solución de problemas reales por tanto se vincula al contexto real donde se generan y se busca solución a dichos problemas interactuando no solo con los docentes, sino además con empresarios, líderes comunitarios y pobladores que serán beneficiados con los resultados obtenidos.

El desarrollo de las actividades en esta dimensión tiene como punto de partida la identificación de problemas reales como la comercialización de productos, creación de pequeñas empresas, elaboración de proyectos, búsqueda de financiamiento, incorporación a niveles de producción y servicio de grupos de atención prioritaria. ${ }^{1}$

La modelación de pequeñas y medianas empresas en la que participan además de los estudiantes y los docentes, futuros empresarios; esta modelación se desarrolla por equipos con la asesoría directa de los docentes de la carrera e implica la elaboración de un informe contentivo de todos los aspectos necesarios para aperturar una empresa bajo las normativas vigentes en Ecuador.

La modelación de procesos dentro de empresas públicas, privadas y mixtas, en las que participan estudiantes, docentes, empresarios y población beneficiada, la misma también se desarrolla de manera colectiva con la asesoría de los docentes y dando cumplimiento a las normativas vigentes en Ecuador.

1 Según la Constitución de la República del Ecuador; Grupos de atención prioritaria: Las personas adultas mayores, niñas, niños y adolescentes, mujeres embarazadas, personas con discapacidad, personas privadas de libertad y quienes adolezcan de enfermedades catastróficas o de alta complejidad, recibirán atención prioritaria y especializada en los ámbitos público y privado. 
Participación en la implementación de los modelos elaborados, una vez elaborados los proyectos los estudiantes bajo la dirección de los docentes involucrados podrán participar en la implementación total o parcial de los proyectos desempeñando funciones de asesores o ejecutores directos.

En suma, el desarrollo de la formación para el emprendimiento en sus tres etapas se despliega en las tres dimensiones señaladas. La interrelación dialéctica al interior de las etapas y dimensiones del proceso de formación para el emprendimiento y entre estas permite la formación del futuro tecnólogo en administración de empresas para el emprendimiento a partir de la apropiación de la competencia emprendimiento. Entre las etapas y dimensiones existen, relaciones dialécticas, todas las etapas del proceso de formación para el emprendimiento se desarrollan en las tres dimensiones, al mismo tiempo al interior de las etapas y las dimensiones existen relaciones de coordinación y complementación.

En las dimensiones extracurricular y socio comunitaria el estudiante tiene la posibilidad de poner en práctica los conocimientos, habilidades y valores adquiridos para resolver problemas profesionales reales o modelados que requieren el desarrollo de investigaciones y la planeación e instrumentación de proyectos innovadores, en las tres dimensiones participan activamente los miembros del ecosistema necesario para la formación de profesionales emprendedores buscando propiciar los ambientes necesarios para el contexto de formación y desempeño emprendedor.

A partir del análisis teórico y empírico que permitieron las relaciones dialógicas anteriores contextualizadas pueden direccionarse acciones concretas para su praxis.

Las relaciones y proceso que se describe, permiten orientar el proceso de formación de la competencia emprendimiento, contribuyendo al perfeccionamiento de la formación y consiguientemente al logro de un desempeño profesional emprendedor desde un emprendimiento humanista y sostenible. Se enfoca desde la educación en valores e involucra a todos los miembros que constituyen el ecosistema necesario para formar profesionales emprendedores.

De igual manera, se considera necesario profundizar en el tema desde la investigación educativa en aspectos como: 
- La modelación del proceso de formación para el emprendimiento.

- La modelación de las potencialidades, el contenido, el lugar y papel de las prácticas pre profesionales en la formación para el emprendimiento.

- Las necesidades y vías de preparación de los miembros de la comunidad educativa con énfasis en los empresarios para desarrollar el proceso de formación para el emprendimiento.

\section{CONCLUSIONES}

Ecuador es un país donde existen jóvenes emprendedores, sin embargo, la educación académica no constituye un eje orientado al mismo, esto hace que gran parte de estos emprendimiento sean desarrollados de forma empírica y con poca probabilidad de éxito. Se plantean estrategias de trabajo conjunto entre el Instituto Superior Tecnológico Bolivariano de Tecnología y los estudiantes a fin de aportar a ellos el conocimiento y el apoyo adecuado para el desarrollo de los diferentes emprendimientos.

Con base en los principios de la concepción pedagógica de formación para el emprendimiento, en ITB, considera que esta formación se desarrolla en diversos contextos formativos dentro y fuera de las instituciones de educación superior en vínculo con el mundo empresarial y a través de la educación en valores y de los métodos de la labor educativa asociados a la misma.

Se propone un proceso de formación en emprendimiento que consiste en la planeación, intervención formativa del emprendimiento, y evaluación de la formación para el emprendimiento, con un enfoque a las competencias, sembrando una jerarquía de valores en correspondencia con el sistema de valores sociales contextualizados que aportan a la apropiación del humanismo emprendedor y sostenible.

La evaluación de la formación para el emprendimiento en ITB, transita por tres dimensiones y contextos en que se desenvuelve el proceso de formación e involucra a toda la comunidad educativa y demás miembros del ecosistema necesario para dicha formación. 


\section{REFERENCIAS}

Bucardo, A., Saavedra, M. L., y Camarena, M. E. (2015). Hacia una comprensión de los conceptos de emprendedores. Suma de Negocios, 98-107.

Chiquito, R., y Corton, B. (2016). Apuntes para una concepción pedagógica para la formación de la competencia para el emprendimiento en los institutos técnicos y tecnológicos de Ecuador. Maestro y Sociedad, 134-146.

DATOSMACRO. (2018). Datosmacro.Com. Recuperado de https://datosmacro.expansion.com/estado/ indice-competitividad-global/ecuador

Echerman, B. (2011). Rumbo a la universidad emprendedora: cambios en la UNIMET. Cuadernos Unimetanos, 6-10.

Lasio, V., Ordeñana, X., Caicedo, G., Sarmiento, A., Y Izquierdo, E. (2018). Global Entrepreneurship Monitor (GEM) 2017. Recuperado de ESPAE-ESPOL: http://espae.espol.edu.ec/wp-content/uploads/documentos/GemEcuador2017.pdf

Nueno, P. (2005). Emprendiendo: hacia el 2010: una renovada perspectiva global del arte de crear empresas y sus artistas. Barcelona, España: Deusto S.A. ediciones.

Samaniego, F. (2011). Analisis y perspectiva del emprendimiento empresarial ecuatoriano en el contexto de la politica economica del buen vivir. Recuperado de EUMED.NET: http://www.eumed.net/cursecon/ecolat/ec/2014/emprendedores.html

Tobon, S. (2013). Formación integral y competencias: Pensamiento Complejo, Currículo, Didáctica y Evaluación. San Diego: Ecoe Ediciones.

Tolozano, R., Ferrer, M., y Forgas, J. (2016). El proyecto de gestión educativa institucional. Un modelo pedagógico para la formación de técnicos y tecnólogos competentes. Santiago, 625-640. 